\title{
Klebsiella Phage KP34 RNA Polymerase and Its Use in RNA Synthesis
}

\author{
Xueling Lu' ${ }^{1}$, Hui Wu ${ }^{1}$, Heng Xia ${ }^{1}$, Fengtao Huang ${ }^{1}$, Yan Yan ${ }^{1}$, Bingbing Yu ${ }^{1}$, Rui Cheng ${ }^{1}$, \\ Zuzanna Drulis-Kawa ${ }^{2}$ and Bin Zhu ${ }^{1 *}$
}

${ }^{1}$ Key Laboratory of Molecular Biophysics, the Ministry of Education, College of Life Sciences and Technology and Shenzhen College, Huazhong University of Science and Technology, Wuhan, China, ${ }^{2}$ Institute of Genetics and Microbiology, University of Wroctaw, Wroctaw, Poland

\section{OPEN ACCESS}

Edited by:

Daniel Yero,

Autonomous University of Barcelona,

Spain

Reviewed by:

Jens Andre Hammerl,

Federal Institute for Risk Assessment

(BfR), Germany

Andrey Shadrin,

Institute of Biochemistry and Physiology of Microorganisms

(RAS), Russia

Silvia Ayora,

Spanish National Research Council

(CSIC), Spain

*Correspondence: Bin Zhu

bin_zhu@hust.edu.cn

Specialty section:

This article was submitted to

Evolutionary and Genomic

Microbiology,

a section of the journal

Frontiers in Microbiology

Received: 15 July 2019

Accepted: 15 October 2019

Published: 31 October 2019

Citation:

LuX, Wu H, Xia H, Huang F, Yan Y, Yu B, Cheng R, Drulis-Kawa Z and Zhu B (2019) Klebsiella Phage KP34 RNA Polymerase and Its Use

in RNA Synthesis.

Front. Microbiol. 10:2487.

doi: 10.3389/fmicb.2019.02487
We have characterized the single subunit RNA polymerase from Klebsiella phage KP34. The enzyme is unique among known bacteriophage RNA polymerases in that it recognizes two unrelated promoter sequences, which provided clues for the evolution of phage single-subunit RNA polymerases. As the first representative enzyme from the "phiKMV-like viruses" cluster, its use in run-off RNA synthesis was investigated. RNASeq analysis revealed that the KP34 RNA polymerase does not possess the undesired self-templated RNA terminus extension known for T7 RNA polymerase and is suitable to synthesize RNAs with structured $3^{\prime}$ termini such as sgRNAs. A KP34 RNA polymerase Y603F mutant is engineered to incorporate deoxy- and $2^{\prime}$-fluoro ribonucleotide into RNA.

Keywords: T7 RNA polymerase, phiKMV, sgRNA, in vitro transcription, run-off RNA synthesis

\section{INTRODUCTION}

In vitro run-off RNA synthesis catalyzed by phage-encoded single-subunit RNA polymerases (ssRNAP) is a fundamental technology for RNA research. The most widely used enzyme in this technique is the RNA polymerase (RNAP) encoded by bacteriophage T7 due to its high efficiency and extensive characterization (Davanloo et al., 1984; Tabor and Richardson, 1985; Milligan and Uhlenbeck, 1989). However, limitations of T7 RNAP such as RNA self-templated extension (Cazenave and Uhlenbeck, 1994; Triana-Alonso et al., 1995; Nacheva and Berzal-Herranz, 2003; Gholamalipour et al., 2018) hinder downstream RNA research and application. Therefore, it is necessary to discover novel ssRNAPs which may overcome limitations of T7 RNAP and improve in vitro transcription technique.

Recently blooming bacteriophage genome resources provide opportunities for new ssRNAPs discovery, and bioinformatics analysis has revealed four distinct phage clusters corresponding to T7-, SP6-, P60-, and phiKMV-like viruses in the short-tailed phage Autographivirinae subfamily (Drulis-Kawa et al., 2011; Figure 1A), which was known to encode ssRNAPs (Supplementary Figure S1). Among them, Enterobacteria phage T7 and T3 from T7-like viruses cluster, Salmonella phage SP6 from SP6-like viruses cluster, and Synechococcus phage Syn5 from P60like viruses cluster have their ssRNAPs and transcription systems characterized (Davanloo et al., 1984; Morris et al., 1986; Krieg and Melton, 1987; Zhu et al., 2013), leaving phiKMVlike viruses the only cluster without any representative ssRNAP of its members studied. 


\section{A}

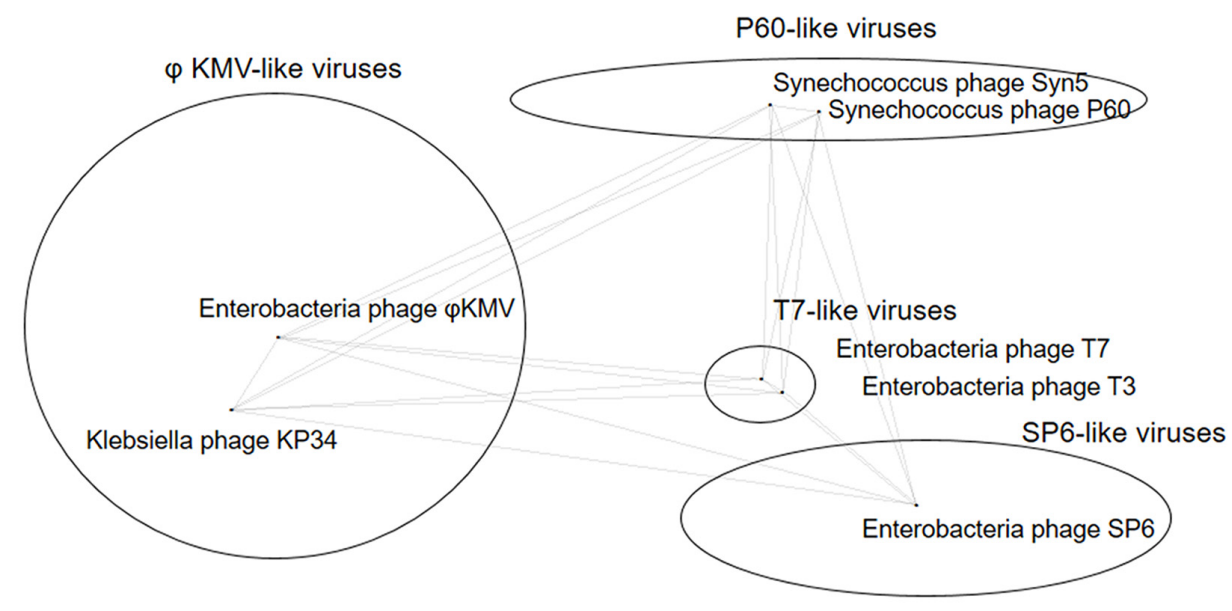

B

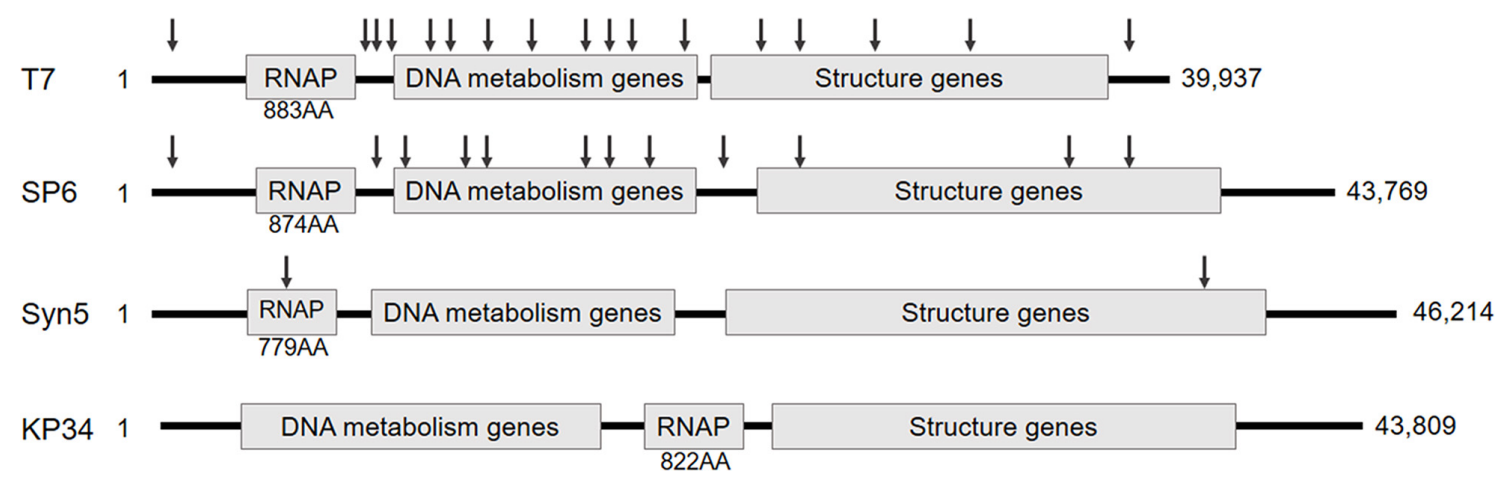

FIGURE 1 | Classification and genome organization of bacteriophages containing ssRNAP. (A) Schematic showing of Autographivirinae clusters based on genome sequence comparison (an inaccurately modified version of Fig. 3 in Drulis-Kawa et al., 2011). (B) Schematic illustration of the genome organization of phage T7, SP6, Syn5 and KP34. The approximate position of T7, SP6, and Syn5 promoters are indicated by down arrows. Size of each RNAP and genome are also shown.

In this work, we targeted Klebsiella phage KP34 to characterize the first ssRNAP from phiKMV-like viruses. Bacteriophage KP34 is a novel virus that propagates on extended-spectrum $\beta$-lactamase-producing Klebsiella pneumoniae strains (DrulisKawa et al., 2011). The genome sequence of phage KP34 consists of 43,809 bp containing 57 predicted ORFs, and the gene that encodes RNAP is between the DNA metabolism genes and the structure genes (Drulis-Kawa et al., 2011). This gene organization is typical among phiKMV-like viruses, while in all other clusters of Autographivirinae, the RNAP gene is located upstream of DNA metabolism genes (Figure 1B) and takes control of the transcription of the latter. The unique gene organization suggests that the ssRNAPs and transcription systems of phiKMV-like viruses are the most distant in evolution from those of the other three categories in the Autographivirinae subfamily, thus the characterization of KP34 RNAP may provide clues for the understanding of the evolution and mechanism of ssRNAP-based transcription systems.

With purified KP34 RNAP, we have established the KP34 in vitro transcription system and identified the KP34 RNAP promoter sequences. Surprisingly, four promoters divided into two unrelated classes were found within the KP34 genome, not known before for any phage ssRNAP transcription system. KP34
RNAP demonstrates its advantage over T7 RNAP in run-off RNA synthesis as it lacks the property of the latter to extend the $3^{\prime}$ primer/template structure of RNA (Gholamalipour et al., 2018), as revealed by RNA-Seq. The Y603F mutant of KP34 RNAP can incorporate dNMPs and 2 '-fluoro-dNMPs into RNA.

\section{MATERIALS AND METHODS}

\section{Materials}

Oligonucleotides were obtained from TsingKe (Beijing, China). DNase I, restriction endonucleases, E. coli inorganic pyrophosphatase, E. coli Poly(A) Polymerase, RNA 5' Pyrophosphohydrolase (RppH), T4 DNA ligase, NTPs, dNTPs, and RNA purification kits were from New England BioLabs (Ipswich, MA, United States). RNase inhibitor was from Thermo Fisher Scientific (Waltham, MA, United States). PrimeSTAR Max DNA Polymerase and Premix Taq DNA Polymerase, SMARTScribe and ProtoScript II Reverse Transcriptase are were from TAKARA (Shiga, Japan). DNA purification kit was from Axygen (Union City, CA, United States). Ni-NTA resin was from Qiagen (Hilden, Germany). Preparative Superdex S200 for gel filtration was from GE Healthcare (Chicago, IL, United States). 
Radiolabeled nucleotides were from PerkinElmer (Waltham, MA, United States). 2'-F-dNTPs were from TriLink (San Diego, CA, United States).

\section{Protein Expression and Purification}

DNA fragments encoding KP34 RNAP were amplified from the KP34 genome and inserted into pET28a vector with N-terminal His-tag. PCR amplification was performed following the user manual of PrimeSTAR Max DNA Polymerase (TAKARA). KP34 genomic DNA was prepared according to a previous report (Drulis-Kawa et al., 2011). The Plasmid pET28a-KP34 RNAP was transformed into E. coli BL21(DE3). The bacteria were cultured in LB medium containing $50 \mu \mathrm{g} / \mathrm{ml}$ kanamycin at $37^{\circ} \mathrm{C}$ until they reached an $A 600$ of $\sim 1.2$. The gene for KP34 RNAP was induced by the addition of $0.5 \mathrm{mM}$ IPTG at $28^{\circ} \mathrm{C}$ and incubation continued for $3 \mathrm{~h}$. Cells were harvested, resuspended and sonicated in lysis buffer $(50 \mathrm{mM}$ Tris- $\mathrm{HCl}, \mathrm{pH} 8.0,300 \mathrm{mM} \mathrm{NaCl}$, and $20 \mathrm{mM}$ imidazole). Resuspended cells were lysed via sonication on ice using $60 \%$ probe amplitude for $5 \mathrm{~min}$ ( $2 \mathrm{~s}$ on; $2 \mathrm{~s}$ off). Cell debris was pelleted by centrifugation for $50 \mathrm{~min}$ at $12000 \mathrm{rpm}, 4^{\circ} \mathrm{C}$. His-tagged KP34 RNAP was purified with Ni-NTA-agarose column. The lysate was run over a $2 \mathrm{ml}$ (bead volume) $\mathrm{Ni}$ NTA gravity column pre-equilibrated with lysis buffer. The column was washed with 10 column volumes of wash buffer (50 mM Tris- $\mathrm{HCl}$, pH 8.0, $300 \mathrm{mM} \mathrm{NaCl}$, and $50 \mathrm{mM}$ imidazole). KP34 RNAP was eluted off the column by 4 column volumes of elution buffer $(50 \mathrm{mM}$ Tris- $\mathrm{HCl}, \mathrm{pH} 8.0$, $300 \mathrm{mM} \mathrm{NaCl}$, and $200 \mathrm{mM}$ imidazole). The RNAP was further purified by gel filtration chromatography on a $200 \mathrm{ml}$ preparative Superdex S200 column. The column was preequilibrated with a $400-500 \mathrm{ml}$ balance buffer $(50 \mathrm{mM}$ Tris$\mathrm{HCl}, \mathrm{pH}$ 8.0, $100 \mathrm{mM} \mathrm{NaCl}, 0.1 \mathrm{mM}$ EDTA and $1 \mathrm{mM}$ DTT). Then a 1-2 $\mathrm{ml}$ sample which was concentrated by Millipore Amicon Ultra-15 (30,000 MWCO) was added to the column. Fractions were eluted off the column by $200-300 \mathrm{ml}$ balance buffer. Fractions containing the KP34 RNAP were concentrated again and dialyzed twice against a storage buffer $(50 \mathrm{mM}$ Tris- $\mathrm{HCl}, \mathrm{pH} 7.5,100 \mathrm{mM} \mathrm{NaCl}, 0.1 \mathrm{mM}$ EDTA, $1 \mathrm{mM}$ DTT, $50 \%$ glycerol, and $0.1 \%$ Triton $^{\text {TM }}$ X-100). KP34 RNAP mutations were introduced through PCR mutagenesis using PrimeSTAR Max DNA Polymerase, and mutant RNAPs were purified following the same procedure as that for the wild-type enzyme. The T7 RNAP gene was cloned into plasmid pQE82L through homologous DNA assembly using ClonExpress ${ }^{\circledR}$ II One Step Cloning Kit from Vazyme (Nanjing, China). The plasmid pQE-82L-T7 RNAP was transformed into BL21(DE3). N-terminal His-tagged T7 RNAP was purified following the same procedure as mentioned above for KP34 RNAP. Histagged Syn5 RNAP was produced from a pET24 vector harboring the N-terminal His-tagged Syn5 RNAP gene between the NdeI and NotI sites and purified according to previous report (Zhu et al., 2015). Oligonucleotide primers used for cloning and mutagenesis are listed in Supplementary Table S1. Protein concentration was determined by the Bradford method, and all RNA polymerases tested in this work were analyzed on SDSPAGE gel (Supplementary Figure S2).

\section{DNA Templates}

Transcription templates for Figures 2C, 3, 5 were prepared by annealing two complementary synthetic DNA oligonucleotides, respectively. Transcription templates for Figure 2A were PCR amplified from the KP34 genomic DNA. To prepare transcription templates for Figure 2D, plasmid pUC19 with a $16 \mathrm{nt}$ or $14 \mathrm{nt}$ KP34 promoter sequence plus three consecutive guanosine residue (in bold) (5'-TAATGTTACAGGAGTAGGG-3' or $5^{\prime}$-ATGTTACAGGAGTAGGG-3') insertion between the BamHI and $X b a I$ sites was linearized by EcoRI treatment. For Figure 4, the coding sequence for an EGFP sgRNA (5'-GGGCACGGGCA GCTTGCCGGGTTTTAGAGCTAGAAATAGCAAGTTAAAAT AAGGCTAGTCCGTTATCAACTTGAAAAAGTGGCACCGAG TCGGTGCTTTTTTTTGAAGAGC-3') under the control of a T7 promoter (5'-TAATACGACTCACTATA-3'), a KP34 promoter ( $5^{\prime}$-TAATGTTACAGGAGTA-3' $)$, or a Syn 5 promoter (5'-ATTGGGCACCCGTAA-3') was inserted into the plasmid pUC19 between the BamHI and XbaI sites, and transcription templates were then PCR amplified from the plasmids carrying each of the three promoters, respectively. Sequences of all PCR primers and synthetic DNA oligonucleotides mentioned above were listed in Supplementary Table S2. PCR reactions for DNA templates were carried out using PrimeSTAR Max DNA Polymerase according to the manual from the manufacturer (TAKARA). PCR products and linearized plasmids were purified using AxyPrep ${ }^{\text {TM }}$ PCR Cleanup Kit (Axygen), and DNA concentrations were determined by NanoPhotometer ${ }^{\circledR}$ (Implen).

\section{Transcription Assays}

For the assays shown in Figures 2A,D, 3A, 4A, 5B,C, the transcription reaction mixtures $(10 \mu \mathrm{l})$ contained $40 \mathrm{mM}$ Tris$\mathrm{HCl}$ (pH 8.0), $2 \mathrm{mM}$ spermidine, $20 \mathrm{mM}$ DTT, $20 \mathrm{mM}$ $\mathrm{MgCl}_{2}, 4 \mathrm{mM}$ of each of the 4 NTPs (with 0 to 4 of the NTPs replaced by their dNTP or 2'-F-dNTP analogs), $40 \mathrm{U} / \mu \mathrm{l}$ RNaseOUT ${ }^{\mathrm{TM}}$ Recombinant Ribonuclease Inhibitor, $0.04 \mathrm{U} / \mu \mathrm{l}$ E. coli inorganic pyrophosphatase, DNA template (2 $\mu \mathrm{M}$ annealed oligonucleotides, $20 \mathrm{nM}$ linearized plasmids, or $0.5 \mu \mathrm{M}$ PCR products), and RNA polymerase (1 $\mu \mathrm{M}$ KP34 RNAP or its mutants, or $0.2 \mu \mathrm{M}$ T7 RNAP or $1 \mu \mathrm{M}$ Syn5 RNAP). Reactions with KP34 and T7 RNAP were carried out at $37^{\circ} \mathrm{C}$ for $1 \mathrm{~h}$ and reactions with Syn5 RNAP were carried out at $24^{\circ} \mathrm{C}$ for $1 \mathrm{~h}$. For the assays shown in Figures $2 \mathrm{~A}$, $\mathbf{3 A}, \mathbf{4 A}$, one unit of DNase I was added to each reaction mixture and incubated for an additional $20 \mathrm{~min}$ at $37^{\circ} \mathrm{C}$ to remove the DNA templates. A total of $1 \mu \mathrm{l}$ of the reaction mixture was then mixed with $9 \mu \mathrm{l}$ deionized $\mathrm{H}_{2} \mathrm{O}$ and $5 \mu \mathrm{l}$ 3X RNA loading dye (95\% formamide, $40 \mathrm{mM}$ EDTA, $0.025 \%$ bromophenol blue and $0.025 \%$ Xylene cyanol FF). Then, $7 \mu \mathrm{l}$ of each sample was loaded onto $12 \%$ TBE native gels or $2 \%$ TAE agarose gels. DNA templates and RNA products were separated by electrophoresis and visualized by staining with ethidium bromide. The transcripts were purified with Monarch ${ }^{\circledR}$ RNA Cleanup Kit (New England BioLabs) and their concentration was determined using NanoPhotometer ${ }^{\circledR}$ (Implen). The reaction mixtures for Figure 2C contained $40 \mathrm{mM}$ Tris- $\mathrm{HCl}$ ( $\mathrm{pH} \mathrm{8.0)}$, $6 \mathrm{mM} \mathrm{MgCl}_{2}, 2 \mathrm{mM}$ spermidine, $10 \mathrm{mM}$ DTT, $200 \mu \mathrm{M} \mathrm{CTP}$, GTP and UTP, $10 \mu \mathrm{M}[\alpha-32 \mathrm{P}] \mathrm{ATP}, 1.5 \mathrm{U} / \mathrm{ml} \mathrm{RNaseOUT}^{\mathrm{TM}}$ 
A

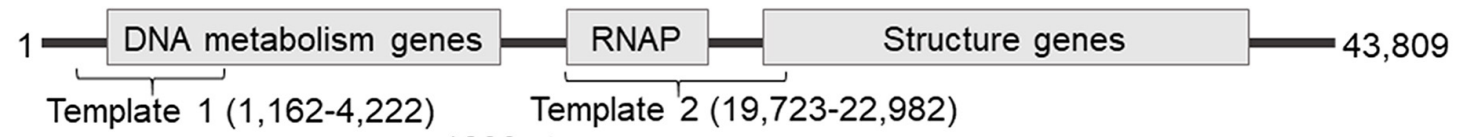

Template $1(1,162-4,222) \quad$ Template $2(19,723-22,982)$

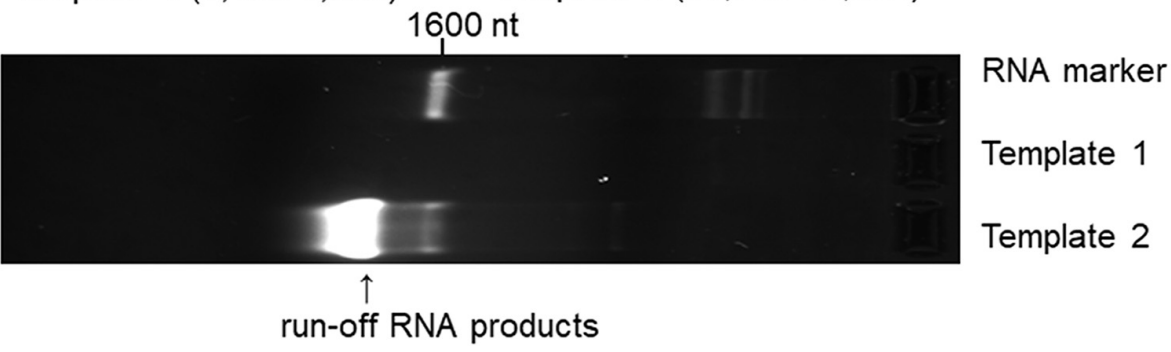

B

AATCCCCGTTCTTCTTCTGC

22,141 GGTACTTTGGACATCCGTCAAGTGCTAG..... TAAAAATTTAATGTTACAGGAGTAGGAATG 22,218

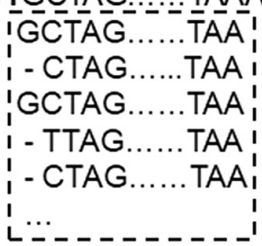

C

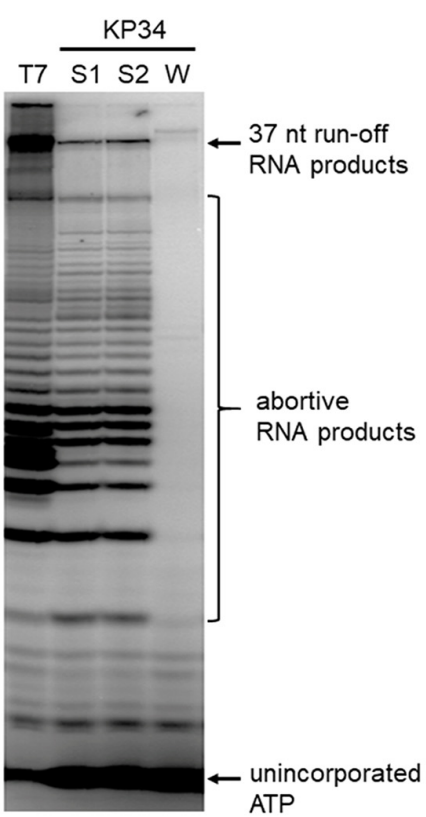

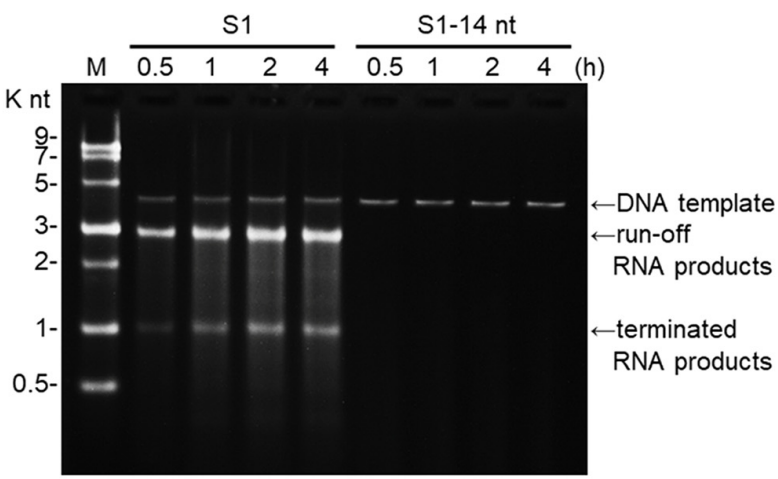

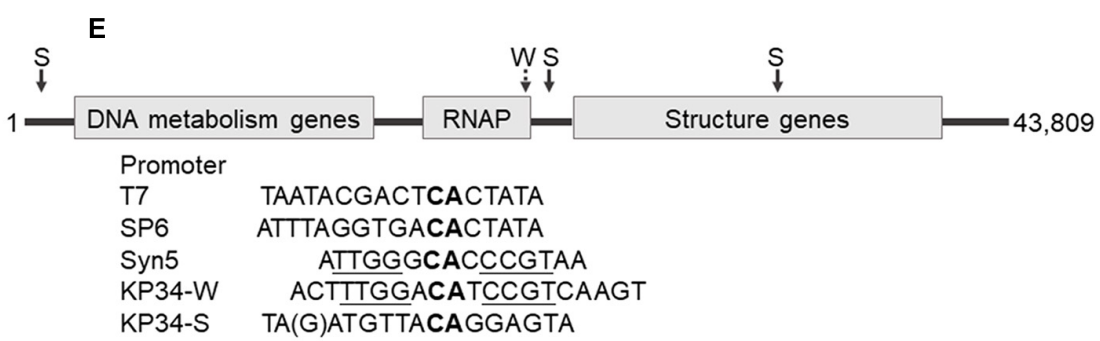

FIGURE 2 | Identification of the KP34 RNAP promoter. (A) The DNA fragment (Template 1) containing previously predicated promoters failed to serve as transcription template for KP34 RNAP to produce RNA in vitro, while the DNA fragment covering the RNAP gene and downstream gap region (Template 2) was active as transcription template. RNA products are shown as bright bands on the $2 \%$ TAE agarose gel. (B) $5^{\prime}$-RACE analysis of the position of transcription initiation. $5^{\prime}$ sequence of KP34 transcripts were matched to KP34 genome. Major sequences were in solid box and minor sequences in dotted box. Their upstream region containing putative promoters is shown in bold. (C) Comparison of run-off RNA synthesis by T7 and KP34 RNAP under the control of various promoters. A DNA template containing a T7 promoter (5'-TAATACGACTCACTATA-3') was incubated with $100 \mathrm{nM}$ T7 RNAP, and three DNA templates containing either a KP34 strong promoter S1 (5'-TAATGTTACAGGAGTA-3'), a KP34 strong promoter S2 (5'-TGATGTTACAGGAGTA-3'), or a KP34 weak promoter W (5'-ACTTTGGACATCCG TCAAGT-3') were incubated with $100 \mathrm{nM} \mathrm{KP34} \mathrm{RNAP} \mathrm{to} \mathrm{direct} \mathrm{to} \mathrm{the} \mathrm{transcription} \mathrm{of} \mathrm{their} \mathrm{downstream} \mathrm{sequence} \mathrm{that} \mathrm{encodes} \mathrm{the} \mathrm{same} 37 \mathrm{nt}$ RNA. [ $\alpha$-32P]ATP was added into reactions for imaging and visualization. Reaction products were separated by a 25\% TBE-Urea denaturing gel. (D) Identification of the full KP34 strong promoter. A KP34 strong promoter S1 (5'-TAATGTTACAGGAGTA-3') or the $3^{\prime} 14 \mathrm{nt}$ common sequence of the two KP34 strong promoters (5'-ATGTTA CAGGAGTA-3') was inserted into plasmid pUC19 to direct the transcription of their downstream sequences, respectively. A run-off transcript of $\sim 2700 \mathrm{nt}$ and a terminated transcript of $\sim 1000 \mathrm{nt}$ (terminated by a predictable T7 class I hairpin terminator structure) were expected from the linearized form of these plasmids if the inserted promoter is sufficient to direct transcription by KP34 RNAP. M: ssRNA Ladder. (E) KP34 promoters in the genome (location of strong promoter (S) pointed by solid arrow and weak promoter (W) by dotted arrow) and comparison of typical ssRNAP promoters. Conserved sequence among ssRNAP promoters are in bold and those homologous between Syn5 promoter and KP34 weak promoter are underlined. 


\section{A 50 nt RNA:}

5'-GCAAAGCUUCGGCUGGUGCAGUGGCCUCAUAAGAGGCGGCCCCUAACAGG-3'

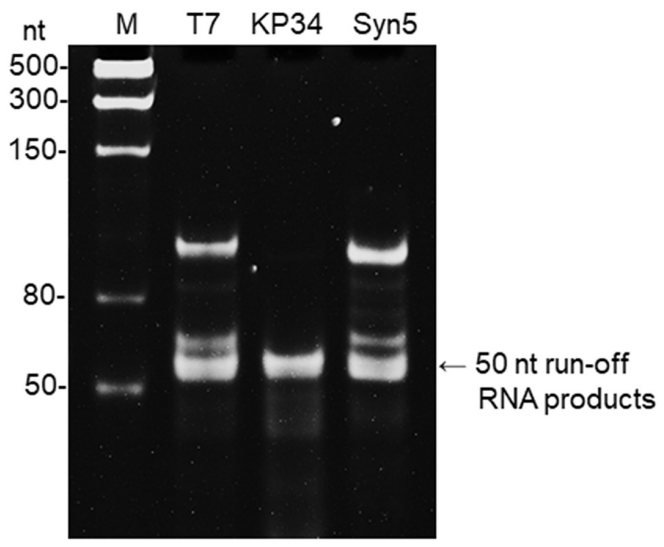

B

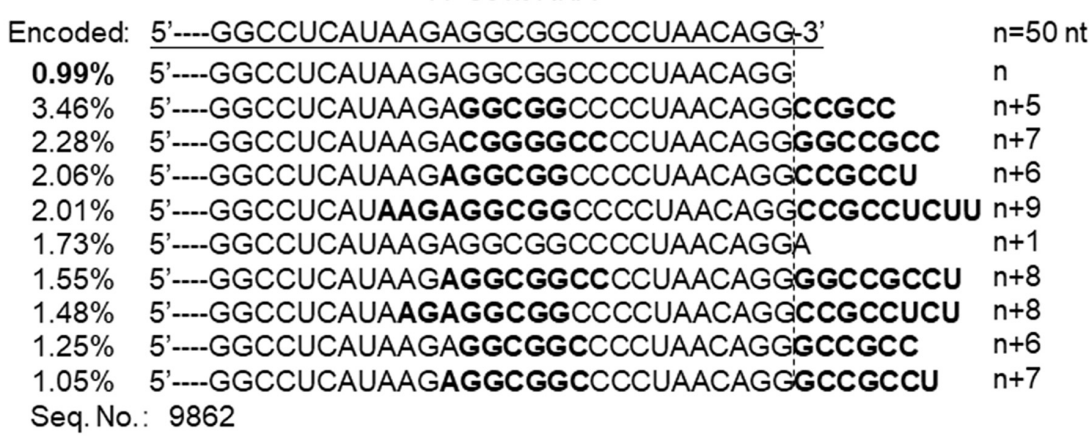

C
KP34-50 ntRNA

Encoded: 5'----GGCCUCAUAAGAGGCGGCCCCUAACAGG-3' n=50 nt

$38.09 \%$ 5'---GGCCUCAUAAGAGGCGGCCCCUAACAGG

$9.16 \%$ 5'---GGCCUCAUAAGAGGCGGCCCCUAAC

$3.04 \%$ 5'---GGCCUCAUAAGAGGCGGCCCCUAACAG

$2.83 \%$ 5'---GGCCUCAUAAGAGGCGGCCCCUAACAGA

$1.34 \%$ 5'---GGCCUCAUAAGAGGCGGCCCCUAACA

Seq. No.: 2303192 $n$

$n-3$

$n-1$

$\underline{\mathrm{n}}$

$\mathrm{n}-2$

FIGURE 3 | Synthesis of a 50 nt RNA containing 3' hairpin structure by various RNAPs. (A) The 50 nt RNA sequence is shown at the top of the gel. The three DNA templates containing the same coding sequences for the $50 \mathrm{nt}$ run-off RNA transcripts under the control of either a T7 promoter, a KP34 strong promoter, or a Syn5 promoter were incubated with $0.2 \mu \mathrm{M}$ T7 RNAP, $1 \mu \mathrm{M} \mathrm{KP34} \mathrm{RNAP,} \mathrm{or} 1 \mu \mathrm{M}$ Syn5 RNAP, respectively. Incubation with KP34 and T7 RNAP was at $37^{\circ} \mathrm{C}$ for $1 \mathrm{~h}$ and incubation with Syn5 RNAP was at $24^{\circ} \mathrm{C}$ for $1 \mathrm{~h}$. Reaction products were separated by a $12 \%$ TBE native gel and then stained with ethidium bromide. M: ssRNA Ladder. (B) RNA-Seq analysis of the $3^{\prime}$ termini of T7 RNAP transcripts. $3^{\prime}$ termini of sequences with reads more than $1 \%$ of total reads were aligned and shown. Percentage of major sequences in total sequencing results are noted and percentage of the correct product is in bold. A dotted line cut indicates the precise terminus encoded by DNA template, and the number of extended $n t$ is shown as $n+x$. Bold sequences indicate complementary sequences in each RNA specie resulted from extension of a possible $3^{\prime}$ self-primed structure. (C) Similar as B, RNA-Seq analysis of the $3^{\prime}$ termini of KP34 RNAP transcripts. Number of missing nt at the $3^{\prime}$ terminus of major sequences is shown as $n-x$.

recombinant ribonuclease inhibitor, $0.5 \mu \mathrm{M}$ DNA templates, and $100 \mathrm{nM}$ KP34 or T7 RNAP that were incubated at $37^{\circ} \mathrm{C}$ for $30 \mathrm{~min}$ before adding one unit of DNase I to each reaction mixture, and then incubated for an additional $20 \mathrm{~min}$ at $37^{\circ} \mathrm{C}$. Reactions were then terminated by the addition of $4 \mu \mathrm{l}$ of $3 \mathrm{X}$ RNA loading dye containing 95\% formamide, $40 \mathrm{mM}$ EDTA, $0.025 \%$ bromophenol blue and $0.025 \%$ Xylene cyanol FF. Samples were then heated at $90^{\circ} \mathrm{C}$ for $1 \mathrm{~min}$, and $4 \mu \mathrm{l}$ of each sample was loaded onto a 25\% TBE-Urea denaturing gel. After electrophoresis, gels were dried and the radioactivity was analyzed using a Fuji BAS 1000 Bioimaging Analyzer.

\section{RNA-Seq}

In vitro transcription products were treated with DNase I for $20 \mathrm{~min}$ at $37^{\circ} \mathrm{C}$ to remove the DNA templates and then were purified with Monarch $^{\circledR}$ RNA Cleanup Kit (New England BioLabs). Transcripts were incubated with RNA 5' Pyrophosphohydrolase $(\mathrm{RppH})$ at $37^{\circ} \mathrm{C}$ for $30 \mathrm{~min}$ to remove 

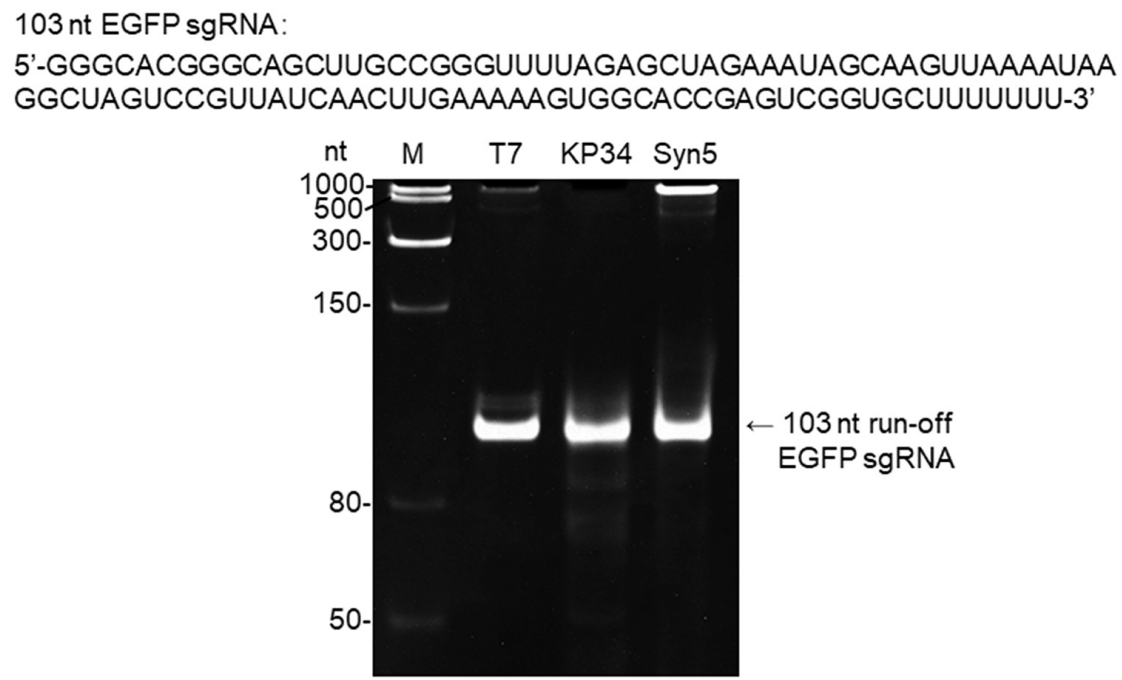

B

T7-103 nt EGFP sgRNA

Encoded: 5'----CGUUAUCAACUUGAAAAAGUGGCACCGAGUCGGUGCUUUUUUU:-3'

1 5'---.CGUUAUCAACUUGAAAAAGUGGCACCGAGUCGGUGCUUUUUUU:

2 5'---CGUUAUCAACUUGAAAAAGUGGCACCGAGUCGGUGCUUUUUUUG

3 5'---.CGUUAUCAACUUGAAAAAGUGGCACCGAGUCGGUGCUUUUUUU!

4 5'---.-CGUUAUCAACUUGAAAAAGUGGCACCGAGUCGGUGCUUUUUUUCCAAGUUG

5 5'---.-CGUUAUCAACUUGAAAAAGUGGCACCGAGUCGGUGCUUUUUUUU

6 5'---.CGUUAUCAACUUGAAAAAGUGGCACCGAGUCGGUGCUUUUUUUंCAAGUUGAUAACG

7 5'---.CGUUAUCAACUUGAAAAAGUGGCACCGAGUCGGUGCUUUUUUUUU

8 5'---.CGUUAUCAACUUGAAAAAGUGGCACCGAGUCGGUGCUUUUUUUCAAGUUG

9 5'---.CGUUAUCAACUUGAAAAAGUGGCACCGAGUCGGUGCUUUUUUU'GAU

10 5'----CGUUAUCAACUUGAAAAAGUGGCACCGAGUCGGUGCUUUUUU

11 5'---.CGUUAUCAACUUGAAAAAGUGGCACCGAGUCGGUGCUUUUUUUCG

12 5'----CGUUAUCAACUUGAAAAAGUGGCACCGAGUCGGUGCUUUUUUUG

$\mathrm{n}=103 \mathrm{nt}$

$n+1$

$n$

$n+7$

$n+1$

$n+13$

$\mathrm{n}+2$

$n+7$

$n+3$

$\mathrm{n}-1$

$\mathrm{n}+2$

$n+1$

C
KP34-103nt EGFP sgRNA

Encoded: 5'----CGUUAUCAACUUGAAAAAGUGGCACCGAGUCGGUGCUUUUUUU-3'

15 '---.-CGUUAUCAACUUGAAAAAGUGGCACCGAGUCGGUGCUUUUUU

2 5'---.CGUUAUCAACUUGAAAAAGUGGCACCGAGUCGGUGCUUUUUUU!

3 5'----CGUUAUCAACUUGAAAAAGUGGCACCGAGUCGGUGCUUUUUUU:

4 5'----CGUUAUCAACUUGAAAAAGUGGCACCGAGUCGGUGCUUUUUU

5 5'---.-CGUUAUCAACUUGAAAAAGUGGCACCGAGUCGGUGCUUUUUUU

6 5'---.CGUUAUCAACUUGAAAAAGUGGCACCGAGUCGGUGCUUUUUU

7 5'---.-CGUUAUCAACUUGAAAAAGUGGCACCGAGUCGGUGCUUUUUU

8 5'----CGUUAUCAACUUGAAAAAGUGGCACCGAGUCGGUGCUUUUUUU:

9 5'---.-CGUUAUCAACUUGAAAAAGUGGCACCGAGUCGGUGCUUUUU

10 5'---.CGUUAUCAACUUGAAAAAGUGGCACCGAGUCGGUGCUUUUUUU:

11 5'----CGUUAUCAACUUGAAAAAGUGGCACCGAGUCGGUGCUUUUUUU:

12 5'----CGUUAUCAACUUGAAAAAGUGGCACCGAGUCGGUGCUUUU

$\mathrm{n}=103 \mathrm{nt}$
$\mathrm{n}-1$
$\mathrm{n}$
$\mathrm{n}$
$\mathrm{n}-1$
$\mathrm{n}$
$\mathrm{n}-1$
$\mathrm{n}-1$
$\mathrm{n}$
$\mathrm{n}-2$
$\mathrm{n}$
$\mathrm{n}$
$\mathrm{n}-3$

$n-3$

FIGURE 4 | Synthesis of an sgRNA by T7 and KP34 RNAP. (A) The sgRNA sequence is shown at the top of the gel. The three DNA templates containing the same coding sequences for the sgRNA under the control of either a T7 promoter, a KP34 strong promoter, or a Syn5 promoter were incubated with $0.2 \mu \mathrm{M}$ T7 RNAP, $1 \mu \mathrm{M}$ KP34 RNAP, or $1 \mu \mathrm{M}$ Syn5 RNAP, respectively. Incubation with KP34 and T7 RNAP was at $37^{\circ} \mathrm{C}$ for $1 \mathrm{~h}$ and incubation with Syn5 RNAP was at $24^{\circ} \mathrm{C}$ for $1 \mathrm{~h}$. Reaction products were separated by a 12\% TBE native gel and then stained with ethidium bromide. M: ssRNA Ladder. (B) 3'-RACE analysis of the 3' termini of T7 RNAP transcripts. $3^{\prime}$ termini of obtained sequences were aligned and shown. A dotted line cut indicates the precise terminus encoded by DNA template and number of extended or missing $n t$ is shown as $n+x$ or $n-x$. Bold sequences indicate complementary sequences in each RNA resulted from extension of possible $3^{\prime}$ self-primed structures. (C) Similar as B, 3'-RACE analysis of the $3^{\prime}$ termini of KP34 RNAP transcripts.

pyrophosphate from the $5^{\prime}$ end of triphosphorylated RNAs and to leave $5^{\prime}$ monophosphate. $5^{\prime}$ monophosphorylated RNAs were purified with Monarch ${ }^{\circledR}$ RNA Cleanup Kit (New England BioLabs), and the cDNA library was prepared by the NEBNext Small RNA Library Prep Set for Illumina (New England BioLabs). The cDNA library was sequenced on an Illumina HiSeq 2500 sequencing platform. The raw data in FASTQ format was obtained from the SeqHealth company (Wuhan, China).

\section{RNA 5' - and $3^{\prime}$-RACE}

Transcription reactions $(10 \mu \mathrm{l})$ were incubated at $37^{\circ} \mathrm{C}$ for 20 min with DNase I to remove the DNA templates. Transcripts 


\section{B 37 nt RNA:}

5'-GGAGAACCUUAAGGUUUAACUUUAAGACCCUUAAGUG-3'

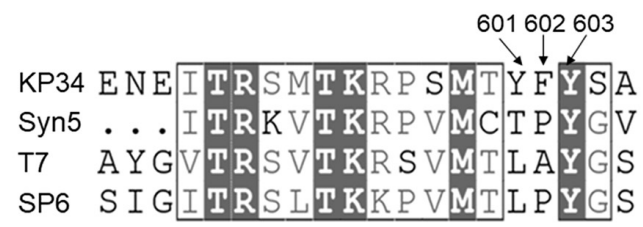

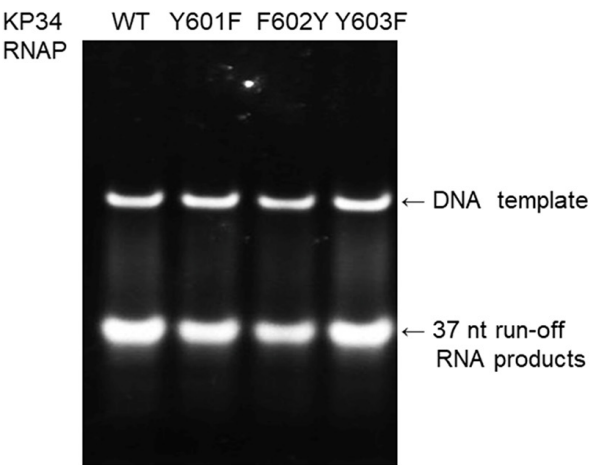

C

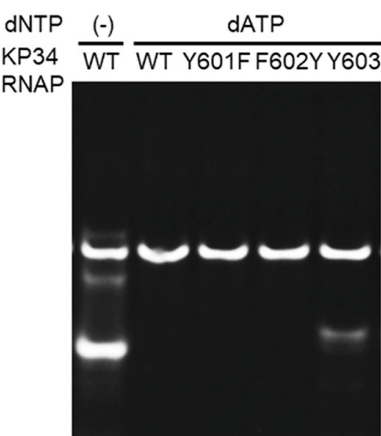

2'-F-dNTP (-) KP34 WT $\overline{\text { WT Y601F F602YY603F WT Y601F F602YY603F }}$ RNAP

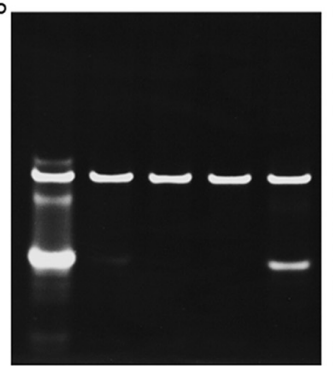

dGTP

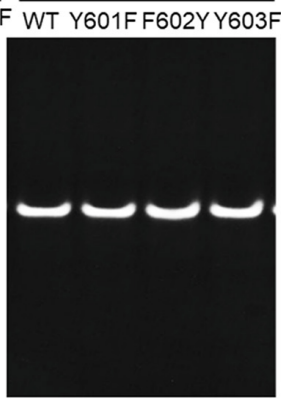

2'-F-dGTP

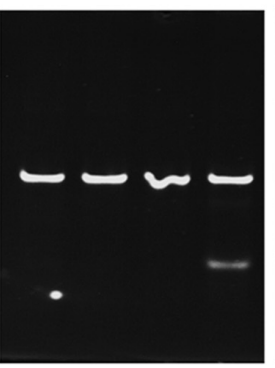

dCTP

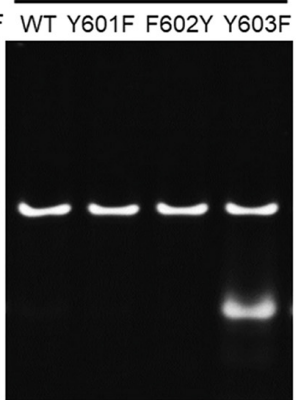

2'-F-dCTP

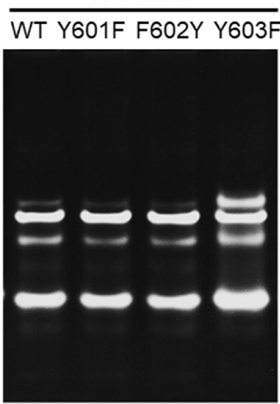

dTTP
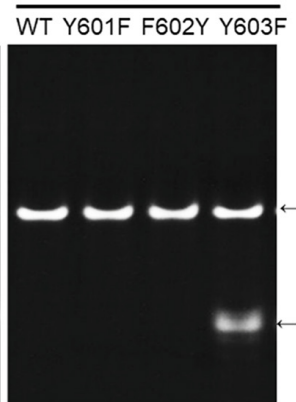

$\leftarrow$ DNA template

37 nt run-off RNA products

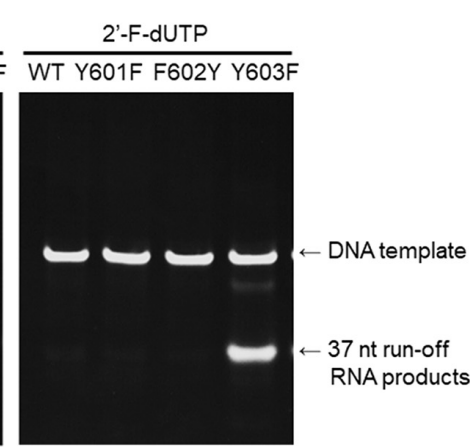

FIGURE 5 | Incorporation of dNMPs and 2'-F-dNMP by wild-type (WT) KP34 RNAP and its Y601F, F602Y, and Y603F mutants. All RNA polymerases in the assays were at the same final concentration $1 \mu \mathrm{M}$. Transcription reaction mixtures were separated by $12 \%$ TBE native gel and then stained with ethidium bromide.

(A) KP34-Y601F, F602Y and KP34-Y603F RNAPs were constructed based on Multiple Sequence Alignment by ClustalW to the region containing either Y639 in T7 RNAP, Y631 in SP6 RNAP or Y564 in Syn5 RNAP, which were known to control the ribose 2' specificity of each enzyme. (B) Comparison of WT, Y601F, F602Y, and Y603F KP34 RNAPs in synthesis of natural RNA. The 37 nt RNA sequence to be synthesized is at the top of the gel. The enzyme used for each reaction is indicated at the top of each lane. (C) Incorporation of dNMPs and 2' -F-dNMPs into the 37 nt RNA transcripts by WT, Y601F, F602Y, and Y603F KP34 RNAPs. In each reaction, one of the four normal NTP substrates was replaced by the corresponding dNTP or $2^{\prime}$-F-dNTP analog as indicated at the top of the gels.

were purified with a Monarch ${ }^{\circledR}$ RNA Cleanup Kit (New England BioLabs) and then incubated with E. coli Poly(A) Polymerase at $37^{\circ} \mathrm{C}$ for $30 \mathrm{~min}$ to add adenosines to the $3^{\prime}$ end of RNA. The products were purified again. RNAs were then reverse transcribed to cDNA.

5'-RACE: $1-3.5 \mu$ l RNA (1 ng-1 $\mu$ g of poly $\mathrm{A}^{+}$RNA) was mixed with $1 \mu 13^{\prime}$ SMART CDS Primer II A $(12 \mu \mathrm{M})$ to $4.5 \mu \mathrm{l}$ total volume. The sample RNA/primer was denatured for $5 \mathrm{~min}$ at $65^{\circ} \mathrm{C}$ and was then spun briefly and put promptly on ice. The following components were added (5.5 $\mu \mathrm{l}): 2 \mu \mathrm{l} 5 \mathrm{X}$ First-Strand Buffer, $0.25 \mu \mathrm{l}$ DTT $(100 \mathrm{mM}), 1 \mu \mathrm{l}$ dNTP Mix (10 mM), $1 \mu \mathrm{l}$ SMARTer II A Oligonucleotide (12 $\mu \mathrm{M}), 0.25 \mu \mathrm{l}$ RNase Inhibitor $(40 \mathrm{U} / \mu \mathrm{l})$, and $1 \mu \mathrm{l}$ SMARTScribe Reverse Transcriptase $(100 \mathrm{U} / \mu \mathrm{l})$. Reaction mixtures were incubated at $42^{\circ} \mathrm{C}$ for $1 \mathrm{~h}$. cDNA amplification was done by $12.5 \mu \mathrm{l}$ Premix Taq DNA Polymerase (TAKARA) using $0.2 \mu \mathrm{M} 5^{\prime}$ universal prime (UPM) and $0.2 \mu \mathrm{M} 3^{\prime}$ gene specific primer (GSP1) in a $25 \mu$ l reaction volume. $3^{\prime}$-RACE: $100 \mathrm{ng}$ poly $\mathrm{A}^{+} \mathrm{RNA}$, 
$5 \mu \mathrm{M} 2 \mu \mathrm{l}$ VN-oligo $(\mathrm{dT})_{18}(50 \mu \mathrm{M})$ was mixed with $1 \mathrm{mM}$ dNTP to $10 \mu \mathrm{l}$ total volume. Sample RNA/primer was denatured for $5 \mathrm{~min}$ at $65^{\circ} \mathrm{C}$, then spun briefly and put promptly on ice. The following components were added $(10 \mu \mathrm{l}): 4 \mu \mathrm{l} 5 \mathrm{X}$ ProtoScript II Buffer, $2 \mu l$ 0.1 M DTT, $1 \mu$ l ProtoScript II Reverse Transcriptase (200 U/ $\mu \mathrm{l}), 0.2 \mu \mathrm{l}$ RNase Inhibitor $(40 \mathrm{U} / \mu \mathrm{l})$, and $2.8 \mu \mathrm{l}$ deionized $\mathrm{H}_{2} \mathrm{O}$. The $20 \mu \mathrm{l} \mathrm{cDNA}$ synthesis reaction was incubated at $42^{\circ} \mathrm{C}$ for $1 \mathrm{~h}$. cDNA was then amplified by $12.5 \mu \mathrm{l}$ Premix Taq DNA Polymerase (TAKARA) using $0.2 \mu \mathrm{M} 5^{\prime}$ gene specific primer (GSP2) and $0.2 \mu \mathrm{M} 3^{\prime} \mathrm{VN}$ primer in a $25 \mu \mathrm{l}$ reaction volume.

PCR products were purified by AxyPrep ${ }^{\text {TM }}$ PCR Cleanup Kit, and then ligated to T-Vector pMD19 with T4 DNA ligase (2X ligation Mix); the reaction was incubated at $16^{\circ} \mathrm{C}$ for $30 \mathrm{~min}$. Plasmids containing inserted sequences were amplified and sent for sequencing.

\section{RESULTS AND DISCUSSION}

\section{Klebsiella Phage KP34 and Its RNAP}

Klebsiella phage KP34 is distantly related to the T7-, SP6and P60-like viruses of the short-tailed phage Autographivirinae subfamily (Figure 1A) and belongs to the cluster of phiKMV-like viruses, which is the only phage cluster in the subfamily without any RNAP of its member characterized. Predicted KP34 RNA polymerase consists of 822 amino acids (AA), which is larger in size than Syn5 RNAP (779 AA) and smaller than T7 (883 AA) and SP6 RNAP (874 AA), and compared to characterized phage ssRNAPs it is the only one with its coding gene located downstream of the DNA metabolism genes (Figure 1B). KP34 RNAP with over $90 \%$ homogeneity was purified as N-terminal His-tagged protein (Supplementary Figure S2).

\section{Identification of the KP34 RNAP Promoter}

Bioinformatics has predicted KP34 transcription promoter to be a consentaneous sequence 5'-AGCCTATA GCGTCCTACGGGGCGCTATGTGAA- $3^{\prime}$ in the early region of the phage genome (Eriksson et al., 2015), because it is the only sequence that is longer than $20 \mathrm{nt}$ and appears twice within the genome. However, a DNA fragment (template 1) made by PCR amplification of this region failed to serve as the template for transcription by KP34 RNAP to produce any RNA product in vitro (Figure 2A).

Deduced from the promoter distribution of known phage ssRNAP promoters (Figure 1B), we predicted that there should be at least one KP34 promoter in the region covering the RNAP gene [as is the case for Syn5 (Zhu et al., 2013)] and the intergenic region between the RNAP and the next gene [as the cases for T7 and SP6 (Davanloo et al., 1984; Krieg and Melton, 1987; Chen and Schneider, 2005)]. Indeed, DNA template 2 made accordingly directed the production of RNA transcripts by KP34 RNAP (Figure 2A). $5^{\prime}$-RACE revealed that most of these transcripts initiated at nucleotide (nt) 22213 in the genome (Figure 2B), strongly suggesting that its upstream region, right behind the RNAP gene (nt 19723-22191), contains the major KP34 promoter. A $16 \mathrm{nt}$ sequence $5^{\prime}$-TAATGTTACAGGAGTA- $3^{\prime}$ was identified in this region by sequence homology analysis; it appears twice in KP34 genome (nt 22197-22212, 33721-33736) and differs with another sequence $5^{\prime}$-TGATGTTACAGGAGTA-3' (475-490) by only the $5^{\prime}$ second nt. In vitro transcription assay confirmed that these two $16 \mathrm{nt}$ sequences promote KP34 RNAP transcription at similar efficiency (Figure 2C, S1 vs. S2), while the common 14 nt sequence of these two is much less efficient as KP34 promoter (Figure 2D). Thus $5^{\prime}$ T(A/G)ATGTTACAGGAGTA-3' was designated as KP34 strong promoter. To our surprise, $5^{\prime}$-RACE also identified a small portion of transcripts not initiated by the strong promoter. These RNA $5^{\prime}$ sequences match the $3^{\prime}$ region $(\sim n t 22164)$ of a putative proximal weak promoter within the RNAP gene (Figure 2B). The $5^{\prime}$ termini of RNA from this promoter show high heterogeneity (Figure 2B), indicating that it is an inefficient and inaccurate promoter. In vitro transcription assay confirmed that the upstream region containing the sequence 5'-ACTTTGGACATCCGTCAAGT-3' (nt 22144-22163) also initiates KP34 RNAP transcription, while with much less efficiency and precision, as judged by the amount and heterogeneity of run-off products (Figure 2C, W vs. S1 or S2). Thus, this sequence was designated as KP34 weak promoter. Coincidentally, the Synechococcus phage Syn5 promoter is also located within its RNAP gene and shares sequence similarity with KP34 weak promoter (Figure 2E). Like other phage ssRNAPs, KP34 RNAP also generates large amounts of abortive transcripts ranging from a few to more than $20 \mathrm{nt}$ during transcription, especially under the control of its strong promoter (Figure 2C), likely due to the transition from transcription initiation to elongation. However, it was noticed that the abortive transcript distributions are different between KP34 and T7 RNAP (Figure 2C), of which the underlying mechanism is to be explored.

The whole picture of representative phage ssRNAP systems was filled by the characterization of KP34 RNAP and its promoters (Figures 1B, 2E). Some phages (such as Syn5) retained their major promoter associated with the RNAP gene to fulfill their transcription, while others (such as T7 and SP6) have evolved promoters at multiple genome locations to control transcription in a finer regulated manner. As an intermediate status, KP34 has evolved its specific strong promoter and may rely only on them for transcription control, but its promoter associated with the RNAP gene, which is likely the primitive type, still remains as an evolutionary relic.

To develop the use of KP34 RNAP in vitro transcription, we optimized its reaction condition in preparative scale RNA synthesis (Supplementary Figures S3-S6). The highest yield of run-off RNA in these assays was observed at a temperature of $37^{\circ} \mathrm{C}-42^{\circ} \mathrm{C}$ (Supplementary Figure S3), $\mathrm{pH}$ 7.5-8.5 (Supplementary Figure S4), and a $\mathrm{MgCl}_{2}$ concentration of 10-20 mM (Supplementary Figure S5). $\mathrm{MnCl}_{2}$ with an optimized concentration of $10 \mathrm{mM}$ also supports KP34 RNAP transcription but is less efficient compared to $\mathrm{MgCl}_{2}$ (Supplementary Figure S5). Addition of salts $\mathrm{NaCl}$, 
$\mathrm{KCl}$ or $\mathrm{NHCl}_{4}$ in the reaction all showed inhibitory effect (Supplementary Figure S5). Thus, for convenience we set $37^{\circ} \mathrm{C}, \mathrm{pH} 8.0$ and $20 \mathrm{mM} \mathrm{MgCl}_{2}$ as standard KP34 RNAP reaction conditions.

\section{KP34 RNAP Does Not Catalyze RNA Self-Templated 3' End Extension}

SsRNAPs are fundamental enzymatic tools for the production of RNA for research and application, due to their simplicity and high efficiency, while some of their undesired properties may affect the quality of RNA products and hinder downstream RNA application. For example, the most popular ssRNAP for in vitro transcription-T7 RNAP catalyzes undesired RNA 3' end extension through multiple mechanisms $[\mathrm{N}+1$ (Milligan et al., 1987; Krupp, 1988), template switch (Rong et al., 1998) and self-primed extension (Triana-Alonso et al., 1995; Nacheva and Berzal-Herranz, 2003)]. Recent analysis based on RNASeq clearly demonstrated the severe product $3^{\prime}$ heterogeneity from T7 RNAP reactions caused by self-templated extension on the intermolecular or intramolecular primer/templatelike RNA structures (Gholamalipour et al., 2018). We have encountered the same problem when synthesizing a 50 nt RNA 5'-GCAAAGCUUCGGCUGGUGCAGUGGCC UCAUAAGAGGCGGCCCCUAACAGG-3' derived from an RNA virus genome. Both T7 and Syn 5 RNAP produce highly heterogenous products (Figure 3A). However, the products of KP34 RNAP appear to form a uniform band in the gel (Figure 3A), missing the bands corresponding to products of larger size as those observed with T7 and Syn5 RNAP. To identify the transcription products by T7 and KP34 RNAP, RNA-Seq was performed. In T7 products, RNA with exactly the desired sequence accounts for surprisingly less than 1\% (Figure 3B), while most products (all sequences with reads more than $1 \%$ of total reads were listed in Figure 3B) have their $3^{\prime}$ ends extended with several extra nts that are complementary to part of the RNA inner sequence, indicating that they are formed by the extension of the self-templated terminal structure. However, in major KP34 RNAP products (all sequences with reads more than $1 \%$ of total reads were listed in Figure 3C), $\sim 38 \%$ are correct sequences without any extension at the $3^{\prime}$ ends. On the contrary, most incorrect products are missing 1-3 nt at their $3^{\prime}$ ends. It is not clear yet whether these truncations are caused by the RNAP activity or environmental RNase degradation.

To confirm that KP34 RNAP is advantageous over T7 RNAP for the synthesis of RNA with $3^{\prime}$ secondary structure, we compared the synthesis of another such RNA, the recently widely used single-guide RNA (sgRNA for CRISPR/Cas9 based genome editing) by the two RNAPs. In gel analysis of an sgRNA synthesis, as expected, a product larger than that of the desired size was not observed for KP34 RNAP while T7 and Syn5 RNAP products contain species significantly larger in size, indicating RNA $3^{\prime}$ undesired extension by these RNAPs and further formation of complex structures by the extended RNA (Figure 4A). Further verified by $3^{\prime}$-RACE, various $3^{\prime}$ overextended (up to $13 \mathrm{nts}$ ) RNA species were found in T7 RNAP products (Figure 4B), while only $3^{\prime}$ truncated RNA found in KP34 products (Figure 4C).
Considering there are several protecting nts at the $3^{\prime}$ end of sgRNA, the truncated RNA products should interfere with genome editing application less than the overextended products since the overextension may disturb the functional structure at the $3^{\prime}$ end of sgRNA, thus KP34 is especially suitable to synthesize sgRNA.

\section{Incorporation of dNMPs and 2' -Fluoro-dNMPs (2' -F-dNMP) by KP34 RNAP and Its Mutants}

RNA containing ribose modifications such as $2^{\prime}-\mathrm{F}$ is more resistant to RNase A, the most common RNase contaminant, which recognizes the $2^{\prime}-\mathrm{OH}$ of pyrimidines for cleavage, resulting in a longer survival time of $2^{\prime}$-F-RNA in vitro and in vivo (Pieken et al., 1991; Ono et al., 1997; Sabahi et al., 2001; Pallan et al., 2011). RNAPs usually prefer NTPs strongly against dNTPs and 2'-FdNTPs; however, replacement of a tyrosine with a phenylalanine in the "O-helix" region such as those in the T7 Y639F (Sousa and Padilla, 1995; Padilla and Sousa, 1999) and Syn5 Y564F (Zhu et al., 2015) RNAP mutants can weaken this discrimination and allow synthesis of partially modified 2 '-F-RNA.

To broaden the application of KP34 RNAP, we investigated the effect of such mutations in the enzyme. Sequence alignment of the KP34 RNAP, Syn5 RNAP, T7 RNAP, and SP6 RNAP was performed with Clustal Omega (Sievers et al., 2011). The alignment was processed for publication using the ESPRIPT server v 3.0 (Robert and Gouet, 2014; Supplementary Figure S1). Interestingly, KP34 RNAP possesses three consecutive aromatic amino acids Y601/F602/Y603 at the homologous position (Figure 5A), unique among known ssRNAPs. Correspondingly, we constructed Y601F, F602Y, and Y603F mutants of KP34 RNAP and obtained purified enzymes (Supplementary Figure S2). Tested on the synthesis of a 37 nt RNA, we found that it is the Y603 that plays a significant role in controlling the ribose $2^{\prime}$ specificity of KP34 RNAP, as the Y603F mutant can utilize almost all sets of dNTPs and $2^{\prime}$-F-dNTPs as substrates and incorporate dNMPs and $2^{\prime}$-F-dNMPs into RNA while retaining a similar efficiency for NMP incorporation as the wild-type enzyme (Figures 5B,C). Although no production of RNA was detected with dGTP (likely because G is the initiating $\mathrm{nt})$, the Y603F can even initiate RNA synthesis with $2^{\prime}$-FdGTP (Figure 5C). Except for $2^{\prime}$-F-dCMP, no dNMP or $2^{\prime}$ F-dNMP incorporation was detected for Y601F and F602Y mutants. Both the wild-type and mutant enzymes incorporate 2'-F-dCMP efficiently (Figure 5C). The KP34 RNAP Y603F also incorporates $2^{\prime}$-F-dNMPs into sgRNA, making it suitable for the synthesis of $2^{\prime}$-F-sgRNA (Supplementary Figure S7). It was noticed that upon incorporation of some dNMPs or 2'-F-dNMPs, the mobility of RNA in native gels shifted significantly (Figure 5C and Supplementary Figure S7), which is likely due to the ribose $2^{\prime}$ modification that alters the molecular weight and the structure of the RNA. However, the possibility that the ribose $2^{\prime}$ modifications may also affect the behavior of the KP34 RNA polymerase at the termini of its transcripts, for example, inducing $3^{\prime}$ extension, remains to be clarified. 


\section{DATA AVAILABILITY STATEMENT}

RNA sequencing data have been deposited in the Sequence Read Archive (SRA) (http://www.ncbi.nlm.nih.gov/sra) with the BioProject accession code PRJNA555705.

\section{AUTHOR CONTRIBUTIONS}

$\mathrm{XL}$ and $\mathrm{BZ}$ designed and performed the experiments and wrote the manuscript. HW and $\mathrm{HX}$ analyzed the data. $\mathrm{FH}$ guided protein expression and purification. YY, BY, RC, and ZD-K provided reagents and contributed to manuscript revision.

\section{REFERENCES}

Cazenave, C., and Uhlenbeck, O. C. (1994). RNA template-directed RNA synthesis by T7 RNA polymerase. Proc. Natl. Acad. Sci. U.S.A. 91, 6972-6976. doi: 10 1073/pnas.91.15.6972

Chen, Z., and Schneider, T. D. (2005). Information theory based T7-like promoter models: classification of bacteriophages and differential evolution of promoters and their polymerases. Nucleic Acids Res. 33, 6172-6187. doi: 10.1093/nar/ gki915

Davanloo, P., Rosenberg, A. H., Dunn, J. J., and Studier, F. W. (1984). Cloning and expression of the gene for bacteriophage T7 RNA polymerase. Proc. Natl. Acad. Sci. U.S.A. 81, 2035-2039. doi: 10.1073/pnas.81.7.2035

Drulis-Kawa, Z., Mackiewicz, P., Kesik-Szeloch, A., Maciaszczyk-Dziubinska, E., Weber-Dabrowska, B., Dorotkiewicz-Jach, A., et al. (2011). Isolation and characterisation of KP34-a novel phiKMV-like bacteriophage for Klebsiella pneumoniae. Appl. Microbiol. Biotechnol. 90, 1333-1345. doi: 10.1007/s00253011-3149-y

Eriksson, H., Maciejewska, B., Latka, A., Majkowska-Skrobek, G., Hellstrand M., Melefors, O., et al. (2015). A suggested new bacteriophage genus, "Kp34likevirus", within the Autographivirinae subfamily of Podoviridae. Viruses 7, 1804-1822. doi: 10.3390/v7041804

Gholamalipour, Y., Karunanayake Mudiyanselage, A., and Martin, C. T. (2018). 3' end additions by T7 RNA polymerase are RNA self-templated, distributive and diverse in character-RNA-Seq analyses. Nucleic Acids Res. 46, 9253-9263. doi: 10.1093/nar/gky796

Krieg, P. A., and Melton, D. A. (1987). In vitro RNA synthesis with SP6 RNA polymerase. Methods Enzymol. 155, 397-415. doi: 10.1016/0076-6879(87) 55027-3

Krupp, G. (1988). RNA synthesis: strategies for the use of bacteriophage RNA polymerases. Gene 72, 75-89. doi: 10.1016/0378-1119(88)90129-1

Milligan, J. F., Groebe, D. R., Witherell, G. W., and Uhlenbeck, O. C. (1987) Oligoribonucleotide synthesis using T7 RNA polymerase and synthetic DNA templates. Nucleic Acids Res. 15, 8783-8798. doi: 10.1093/nar/15.21.8783

Milligan, J. F., and Uhlenbeck, O. C. (1989). Synthesis of small RNAs using T7 RNA polymerase. Methods Enzymol. 180, 51-62. doi: 10.1016/0076-6879(89)800916

Morris, C. E., Klement, J. F., and McAllister, W. T. (1986). Cloning and expression of the bacteriophage T3 RNA polymerase gene. Gene 41, 193-200. doi: 10.1016 0378-1119(86)90098-3

Nacheva, G. A., and Berzal-Herranz, A. (2003). Preventing nondesired RNAprimed RNA extension catalyzed by T7 RNA polymerase. J. Biol. Chem. 270 1458-1465. doi: 10.1046/j.1432-1033.2003.03510.x

Ono, T., Scalf, M., and Smith, L. M. (1997). 2'-Fluoro modified nucleic acids: polymerase-directed synthesis, properties and stability to analysis by matrixassisted laser desorption/ionization mass spectrometry. Nucleic Acids Res. 25 4581-4588. doi: 10.1093/nar/25.22.4581

Padilla, R., and Sousa, R. (1999). Efficient synthesis of nucleic acids heavily modified with non-canonical ribose 2'-groups using a mutant T7 RNA

\section{FUNDING}

This project was funded by the National Natural Science Foundation of China (Grants 31670175 and 31870165) and Shenzhen Science and Technology Innovation Fund (Grant JCYJ20170413115637100).

\section{SUPPLEMENTARY MATERIAL}

The Supplementary Material for this article can be found online at: https://www.frontiersin.org/articles/10.3389/fmicb. 2019.02487/full\#supplementary-material

polymerase (RNAP). Nucleic Acids Res. 27, 1561-1563. doi: 10.1093/nar/27.6. 1561

Pallan, P. S., Greene, E. M., Jicman, P. A., Pandey, R. K., Manoharan, M., Rozners, E., et al. (2011). Unexpected origins of the enhanced pairing affinity of 2'-fluoromodified RNA. Nucleic Acids Res. 39, 3482-3495. doi: 10.1093/nar/gkq1270

Pieken, W. A., Olsen, D. B., Benseler, F., Aurup, H., and Eckstein, F. (1991). Kinetic characterization of ribonuclease-resistant 2'-modified hammerhead ribozymes. Science 253, 314-317. doi: 10.1126/science.1857967

Robert, X., and Gouet, P. (2014). Deciphering key features in protein structures with the new ENDscript server. Nucleic Acids Res. 42, 320-324. doi: 10.1093/ nar/gku316

Rong, M., Durbin, R. K., and McAllister, W. T. (1998). Template strand switching by T7 RNA polymerase. J. Biol. Chem. 273, 10253-10260. doi: 10.1074/jbc.273. 17.10253

Sabahi, A., Guidry, J., Inamati, G. B., Manoharan, M., and Wittung-Stafshede, P. (2001). Hybridization of 2'-ribose modified mixed-sequence oligonucleotides: thermodynamic and kinetic studies. Nucleic Acids Res. 29, 2163-2170. doi $10.1093 / \mathrm{nar} / 29.10 .2163$

Sievers, F., Wilm, A., Dineen, D., Gibson, T. J., Karplus, K., Li, W., et al. (2011). Fast, scalable generation of high-quality protein multiple sequence alignments using Clustal omega. Mol Syst Biol. 7, 1-6. doi: 10.1038/msb.2011.75

Sousa, R., and Padilla, R. (1995). A mutant T7 RNA polymerase as a DNA polymerase. EMBO J. 14, 4609-4621. doi: 10.1002/j.1460-2075.1995.tb00140.x

Tabor, S., and Richardson, C. C. (1985). A bacteriophage T7 RNA polymerase/promoter system for controlled exclusive expression of specific genes. Proc. Natl. Acad. Sci. U.S.A. 82, 1074-1078. doi: 10.1073/pnas.82.4.1074

Triana-Alonso, F. J., Dabrowski, M., Wadzack, J., and Nierhaus, K. H. (1995). Selfcoded 3'-extension of run-off transcripts produces aberrant products during in vitro transcription with T7 RNA polymerase. J. Biol. Chem. 270, 6298-6307. doi: 10.1074/jbc.270.11.6298

Zhu, B., Hernandez, A., Tan, M., Wollenhaupt, J., Tabor, S., and Richardson, C. C. (2015). Synthesis of 2'-Fluoro RNA by Syn5 RNA polymerase. Nucleic Acids Res. 43, e94. doi: 10.1093/nar/gkv367

Zhu, B., Tabor, S., Raytcheva, D. A., Hernandez, A., King, J. A., and Richardson, C. C. (2013). The RNA polymerase of marine cyanophage Syn5. J. Biol. Chem. 288, 3545-3552. doi: 10.1074/jbc.M112.442350

Conflict of Interest: The authors declare that the research was conducted in the absence of any commercial or financial relationships that could be construed as a potential conflict of interest.

Copyright (C) 2019 Lu, Wu, Xia, Huang, Yan, Yu, Cheng, Drulis-Kawa and Zhu. This is an open-access article distributed under the terms of the Creative Commons Attribution License (CC BY). The use, distribution or reproduction in other forums is permitted, provided the original author(s) and the copyright owner(s) are credited and that the original publication in this journal is cited, in accordance with accepted academic practice. No use, distribution or reproduction is permitted which does not comply with these terms. 